\title{
Rituximab therapy for juvenile-onset systemic lupus erythematosus
}

\author{
Obioma Nwobi • Carolyn L. Abitbol • \\ Jayanthi Chandar • Wacharee Seeherunvong • \\ Gastón Zilleruelo
}

Received: 12 July 2007 /Revised: 15 October 2007 / Accepted: 24 October 2007 / Published online: 19 December 2007

(C) IPNA 2007

\begin{abstract}
Rituximab (RTX), an anti-CD20 monoclonal antibody, has been proposed for use in the therapy of systemic lupus erythematosus (SLE). We present the initial long-term experience of the safety and efficacy of rituximab for treatment of SLE in children. Eighteen patients (mean age $14 \pm 3$ years) with severe SLE were treated with rituximab after demonstrating resistance or toxicity to conventional regimens. There was a predominance of female (16/18) and ethnic African (13/18) patients. All had lupus nephritis [World Health Organization (WHO) classes 3-5] and systemic manifestations of vasculitis. Clinical disease activity of the SLE was scored with the SLE-disease activity index 2K (SLEDAI-2K). Patients were followed-up for an average of $3.0 \pm 1.3$ years (range 0.5 to 4.8 years). B-cell depletion occurred within 2 weeks in all patients and persisted for up to 1 year in some. Clinical activity scores, double-stranded DNA (dsDNA) antibodies, renal function and proteinuria [urine protein to creatinine ratio (Upr/cr)] improved in $93 \%$ of the patients. Five patients required multiple courses of RTX for relapse, with B-cell repopulation. One died of infectious endocarditis related to severe immunosuppression. In conclusion, our data support the efficacy of rituximab as adjunctive treatment for SLE in children. Although rituximab was well tolerated by the majority of patients, randomized controlled trials are required to establish its long-term safety and efficacy.
\end{abstract}

The authors declare that they have no conflict of interest.

O. Nwobi • C. L. Abitbol $(\triangle) \cdot J$. Chandar $\cdot$ W. Seeherunvong

G. Zilleruelo

Division of Pediatric Nephrology, Department of Pediatrics,

University of Miami/Holtz Children's Hospital,

1611 NW 12th Avenue, Annex 5,

Miami, FL 33126, USA

e-mail: cabitbol@med.miami.edu
Keywords Rituximab - Systemic lupus erythematosus . Children

\section{Introduction}

Systemic lupus erythematosus (SLE) in children is known to carry a worse prognosis than in adults, especially in those of African and Hispanic ethnicity [1, 2]. Although treatment protocols for proliferative lupus nephritis have evolved during the past two decades, pediatric regimens have been center based and selectively derived from adult protocols [3]. These protocols primarily include cyclophosphamide (CYC), which carries long-term toxicities related to malignancy and gonadal dysfunction [4].

Most recently, B-lymphocyte function has been recognized as a major component in the pathogenesis of autoimmune diseases [5]. Rituximab (RTX), a chimeric monoclonal antibody directed against CD20 lymphocytes (commonly called B-cells), was developed as a primary treatment against B-cell lymphoma [6] and is now approved for use in the treatment of rheumatoid arthritis [7]. Although clinical trials of the use of RTX in adults with SLE are substantial and ongoing [8], studies in children have been limited $[9,10]$. A collaborative retrospective and uncontrolled multicenter experience from Europe reported mixed results [11]. Unfortunately, that study failed to use a single protocol, and many patients were treated concurrently with more toxic medications, which made safety analysis unreliable [11].

The purpose of our study was to determine the safety and efficacy of RTX therapy in the treatment of children with severe SLE. Over the course of 7 years, we retrospectively assessed the indications and responses to RTX therapy in a cohort of children with active SLE that 
was refractory to conventional therapies or who had clinical indications prohibiting the use of more toxic immunosuppressant drugs.

\section{Patients and methods}

A retrospective analysis was performed on a cohort of 51 patients diagnosed with SLE and lupus nephritis who received their care at Holtz Children's Hospital, University of Miami Miller School of Medicine, between January 1996 and June 2007. The study was approved by the institutional review board, with waiver of consent authorization, and all subjects were assured anonymity in compliance with the Health Insurance Portability and Accountability Act (HIPAA). The medical records were reviewed for patients' demographic characteristics, age at diagnosis, prior and current medical treatments, type of lupus nephritis and serologic and clinical response to therapy.

Children were considered eligible for inclusion in the analysis if they fulfilled the following criteria: (1) American College of Rheumatology (ACR) criteria for the diagnosis of SLE [12]; (2) age < 16 years at the time of diagnosis of SLE; (3) treatment with the anti-CD20 monoclonal antibody, rituximab, during the observation period from January 2000 through October 2007.

\section{Clinical protocol}

Rituximab (RTX) was administered weekly for two to four doses. The initial dose was $188 \mathrm{mg} / \mathrm{m}^{2}$, infused over $4 \mathrm{~h}$. Subsequent doses were $375 \mathrm{mg} / \mathrm{m}^{2}$ per dose, infused over $6 \mathrm{~h}$ to $8 \mathrm{~h}$. Premedication initially consisted of diphenhydramine and acetaminophen, administered 30 min before the rituximab infusion. However, mild infusion reactions, including pruritis, nausea and emesis, resulted in our adding hydrocortisone $50-100 \mathrm{mg}$ intravenously $30 \mathrm{~min}$ prior to each infusion. Lymphocyte subsets were assayed before and after each course of RTX therapy at 1- to 3-month intervals. This included B cells assayed as CD19+ lymphocytes and T-cells assayed as CD4 and CD8 lymphocytes as total and percent of the total lymphocyte cell count. The serum immunoglobulin levels were measured every 1 to 6 months prior to and after the course of RTX. Adverse events were recorded from the time of the first infusion until 1 year following the treatment course.

Lupus disease activity index and serology

Clinical and biological parameters were assessed prior to the RTX infusion course, at 1- to 3-month intervals thereafter. The clinical disease activity was scored with the SLE-disease activity index $2 \mathrm{~K}$ (SLEDAI-2K), which has a maximum activity score of 105 [13]. Laboratory evaluations included serum complement components (C3 and C4) and anti-double stranded DNA (Anti-dsDNA) antibody and anti-nuclear antibody (ANA).

Lupus nephritis and renal disease activity

Renal biopsies were classified according to the new proposed modifications of the World Health Organization (WHO) classifications [14]. Three patients were treated at the time they were on hemodialysis for extra-renal manifestations of SLE. For those patients not on dialysis, degree of proteinuria and renal function were assessed in response to RTX therapy. Degree of proteinuria was determined by the random urine protein to creatinine ratio (Upr/cr) in milligrams per milligram $(\mathrm{mg} / \mathrm{mg})$, with normal $<0.2$ and nephrotic range $>1.0[15,16]$. Serum albumin (Salb) was assayed as an indirect measure of the nephrotic syndrome. Serum creatinine (Scr) was assayed, and an estimation of glomerular filtration rate (eGFR) was derived by the height index formula of Schwartz, expressed as milliliters per minute per 1.73 meters squared $(\mathrm{ml} / \mathrm{min}$ per $1.73 \mathrm{~m}^{2}$ ) [17]. Complete renal remission was defined as normalization of $\mathrm{Upr} / \mathrm{cr}$ and eGFR. Partial remission was defined as $>50 \%$ decline in Upr/cr. All serum and urine chemical analyses and immunoassays were performed in the central hospital clinical laboratory or the Quest referral laboratories.

\section{Statistical methods}

All data sets were analyzed for Gaussian distribution using the D'Agostino-Pearson omnibus test for normality. The data were calculated into average values at baseline and at 2 months, 4 months, and 6 months after initiation of treatment. Variables at baseline, during and after the treatment were compared by the Wilcoxon matched-pairs signed-ranks test. Differences in parameters between the 2 independent groups were analyzed using Mann-Whitney and the Friedman test with Dunn's multiple comparisons post tests for repeated measures of nonparametric data. $P$ values of less than 0.05 were considered significant. All results are expressed as mean \pm standard deviation (SD).

\section{Results}

Patients' demographics

During the period of observation 18 patients were treated with RTX for at least one course of two to four doses. 
There were 16 female patients and two male patients. Mean age at the time of diagnosis of SLE was $10.7 \pm 2.5$ years (range 7 to 14 years). Mean duration of disease prior to treatment with RTX was $3.1 \pm 2.5$ years (range 2 months to 8 years). Age at the time of first RTX treatment was $14.2 \pm$ 3.3 years, and current age at the time of this report was $17 \pm$ 5.0 years.

All patients had clinical evidence of lupus nephritis. Individual patient demographics, clinical manifestations of SLE and indications for rituximab therapy for patients not on hemodialysis are shown in Table 1. All patients received concurrent therapy with low-dose corticosteroids (CSs) and hydroxychloroquine (HCQ). Sixteen patients had failed or had suffered toxic effects from multiple courses of intravenous therapy with cyclophosphamide (CYC) and corticosteroids. Treatment with RTX included concurrent or subsequent treatment with maintenance doses of mycophenolate mofetil (MMF) or azathioprine (AZT) similar to recently published adult protocols [18].

All three patients on hemodialysis responded positively to the RTX therapy. Patient HD-1 had Raynaud's syndrome and finger necrosis; her symptoms resolved, and she recently received a transplant, 4 years after RTX treatment. Patient HD-2, who had been on ventilator support with pneumonitis, pleuritis and carditis for 1 month prior to receiving RTX, recovered and has been stable on hemodialysis as an outpatient for $>4$ years since RTX therapy. Patient HD-3, after 3 years on hemodialysis, developed debilitating chorea with increased lupus serology. She did not respond to anti-convulsant therapy. The chorea resolved within weeks of the RTX therapy. She remains on dialysis without recurrence of chorea.
Table 1 Patients' baseline characteristics, indications for rituximab treatment, and clinical course ( $F$ female, $M$ male, $H$ Hispanic, $B$ Black, $H T$ hypertension, $C Y C$ cyclophosphamide, $C y A$ cyclosporine A, AZT azathioprine, $M M F$ mycophenolate mofetil, IVIG intravenous therapy with gamma globulin, $C S$ corticosteroids, $H X Q$ hydroxychloroquine, $F R$ full remission, $P R$ partial remission)

\begin{tabular}{|c|c|c|c|c|}
\hline \multirow{2}{*}{$\begin{array}{l}\text { Nephritis } \\
\text { class }\end{array}$} & \multirow{2}{*}{$\begin{array}{l}\text { Previous and/ } \\
\text { or concurrent } \\
\text { therapies }^{\mathrm{a}}\end{array}$} & \multicolumn{2}{|c|}{$\mathrm{Upr} / \mathrm{cr}(\mathrm{mg} / \mathrm{mg})$} & \multirow[t]{2}{*}{ Outcome } \\
\hline & & $\begin{array}{l}\text { Before } \\
\text { RTX }\end{array}$ & $\begin{array}{l}\text { After } \\
\text { RTX }\end{array}$ & \\
\hline IV & $\mathrm{CYC}, \mathrm{MMF}$ & 13.0 & 0.1 & FR \\
\hline IV & $\mathrm{CYC}, \mathrm{MMF}$ & 2.1 & 0.6 & PR \\
\hline IV & $\mathrm{CYC}, \mathrm{MMF}$ & 4.0 & 1.7 & PR \\
\hline IV-V & CYC, MMF, IVIG & 2.6 & 0.2 & FR \\
\hline IV-V & $\mathrm{CYC}, \mathrm{AZT}$ & 0.4 & 0.1 & FR \\
\hline NA & CYC, AZT, MMF, IVIG & 0.6 & 0.3 & PR \\
\hline IV-V & CYC, AZT, MMF, IVIG & 2.6 & NA & Died \\
\hline IV & CYC, AZT, IVIG & 3.6 & 1.9 & PR \\
\hline III & IVIG & 1.7 & 0.2 & FR \\
\hline IV & CYC, MMF, IVIG & 3.0 & 0.7 & PR \\
\hline $\mathrm{V}$ & MMF & 9.7 & 0.2 & FR \\
\hline IV-V & $\begin{array}{l}\text { CYC, AZT, MMF, IVIG, } \\
\text { СyA }\end{array}$ & 5.4 & 0.2 & FR \\
\hline IV & MMF & 1.5 & 0.2 & FR \\
\hline III & CYC, MMF, IVIG & 2.3 & 0.8 & PR \\
\hline IV & CYC, MMF, IVIG & 6.8 & 0.9 & PR \\
\hline
\end{tabular}

${ }^{\mathrm{a}}$ All patients were treated with CS and HXQ 
B-cell depletion and re-population

Figure 1 shows the average B-cell depletion relative to the beginning of the first dose of RTX. Patients received an average of $4.3 \pm 1.6$ doses overall. B-cell depletion was achieved within 2 weeks of the first dose of RTX and lasted an average of $3 \pm 4$ months (range 3 to 12 months). Five patients (28\%) received repeated courses of RTX therapy ranging from two to four courses of one to four additional doses following re-population of the B-cell line in the setting of clinical relapse of SLE. Overall follow-up period from the initial dose of RTX to this report was $3.0 \pm$ 1.3 years. It should be noted that those patients who did not have clinical relapse were not routinely assayed for lymphocyte subsets. Therefore, it is not known if or when those patients with persistent clinical remission had their Blymphocyte lineage re-populated.

Table 2 describes the changes in the lymphocyte subsets from baseline to the nadir drop in the B-lymphocyte lineage. Total lymphocyte counts, as well as CD4 and CD8 T-cell counts, tended to be lower before RTX treatment and increased after RTX therapy. Similarly, the levels of serum immunoglobulins remained normal in comparison with the pre-RTX values.

Lupus activity and auto-antibody response

Table 2 and Fig. 2 summarize the clinical response to RTX therapy. The average lupus activity score (SLEDAI) improved in all patients. Average dsDNA antibody decreased and serum $\mathrm{C} 3$ complement increased significantly after RTX therapy. Other serological parameters showed a trend towards improvement as well.

\section{B-Cell Depletion}

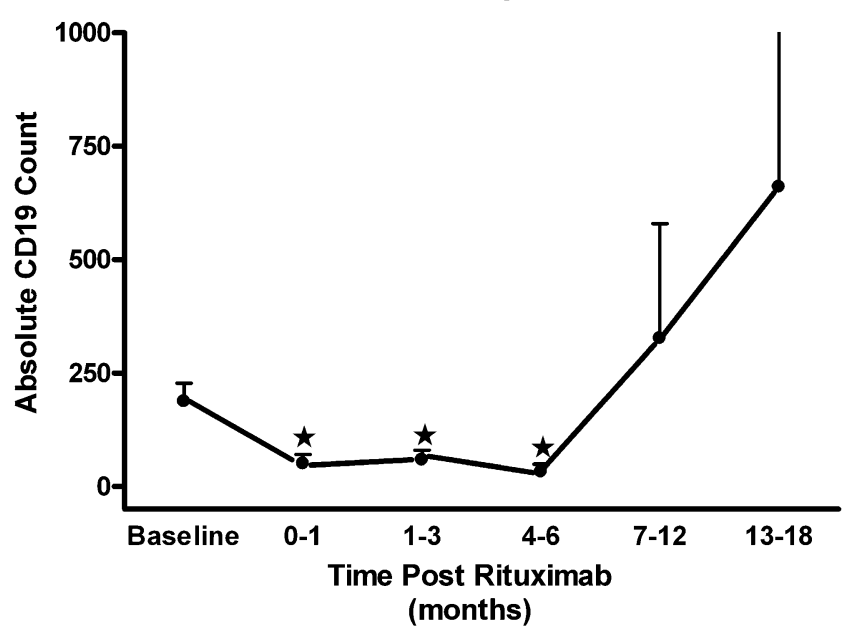

Fig. 1 Graph of B-cell depletion over time from before rituximab therapy to $12-18$ months after rituximab therapy. Asterisks indicate significant difference from the post-rituximab values; $P<0.01$
Table 2 Clinical parameters before and after rituximab therapy (NS not significant)

\begin{tabular}{llll}
\hline Parameter & $\begin{array}{l}\text { Before rituximab } \\
\text { therapy }\end{array}$ & $\begin{array}{l}\text { After rituximab } \\
\text { therapy }\end{array}$ & $P$ \\
\hline Total lymphocyte count & $1044 \pm 596$ & $1734 \pm 1535$ & $\mathrm{NS}$ \\
CD4+ lymphocytes & $344 \pm 217$ & $556 \pm 264$ & $\mathrm{NS}$ \\
CD19+ lymphocytes & $243 \pm 223$ & $74 \pm 71$ & 0.005 \\
Immunoglobulin IgG & $1603 \pm 848$ & $1596 \pm 1055$ & $\mathrm{NS}$ \\
Immunoglobulin IgM & $273 \pm 514$ & $283 \pm 531$ & $\mathrm{NS}$ \\
Upr/cr (mg/mg) & $4.0 \pm 3.5$ & $0.6 \pm 0.6$ & 0.001 \\
Serum albumin (g/dl) & $2.6 \pm 0.7$ & $3.5 \pm 0.6$ & 0.001 \\
Scr (mg/dl) & $1.2 \pm 0.4$ & $0.6 \pm 0.2$ & 0.001 \\
eGFR (ml/min/1.73m $\left.{ }^{2}\right)$ & $86 \pm 32$ & $144 \pm 37$ & 0.0003 \\
Corticosteroid dose & $79 \pm 26$ & $13 \pm 20$ & $<0.0001$ \\
$\quad$ (mg/m ${ }^{2}$ per day) & & & \\
SLEDAI-2K score & $47 \pm 19$ & $25 \pm 14$ & 0.0004 \\
\hline
\end{tabular}

All 15 patients not on dialysis had active lupus nephritis with significant proteinuria. Table 1 provides detailed information regarding the classification of the lupus nephritis for each patient, together with extra-renal manifestations and concurrent or previous therapies, as well as their final outcome. All parameters improved or stabilized in $14 / 15(93 \%)$ patients. Seven had total remission of proteinuria, while the other seven had partial remission, with significant improvement in proteinuria and renal function. Coincident with the improvement in renal disease, the requirement for maintenance corticosteroid therapy was reduced in most patients, with an average fall in corticosteroid dosing from $79 \pm 29 \mathrm{mg} / \mathrm{m}^{2}$ per day before RTX therapy to $13 \pm 20 \mathrm{mg} / \mathrm{m}^{2}$ per day after RTX therapy $(P<0.0001)$.

\section{Adverse events}

One patient (\#7) received three doses of RTX shortly after a cycle of intravenous cyclophosphamide (CYC) administration in an effort to control lupus serositis and nephritis. She developed Staphylococcus aureus endocarditis, with vegetations on the mitral and aortic valves. She died after open heart surgery. This was considered a death from overwhelming infection most likely related to excessive immune suppression. Her CD4 count prior to RTX was $220 / \mathrm{mm}^{3}$, possibly related to prior CYC therapy. At the time of her death her serum immunoglobulin levels were normal.

Patient \#13 had systemic vasculitis and class IV lupus nephritis. Since she was only 7 years of age, it was decided to treat her with intravenous administration of Solu-Medrol followed by weekly RTX for four doses and concurrent mycophenolate mofetil (MMF) as induction therapy. She responded well to RTX, with resolution of her proteinuria and clinical remission of the lupus nephritis. However, she 
Fig. 2 Composite graphs depicting response to rituximab therapy on clinical parameters. Panel a SLEDAI [13] scores before and after rituximab therapy. Panel $\mathbf{b}$ proteinuria (Upr/cr) before and after rituximab. Panel c auto-antibody titers before and after rituximab. Panel d serum complements as $\mathrm{C} 3$ and C4 complements. Asterisks indicate significant differences from the post-rituximab values; $P<0.01$
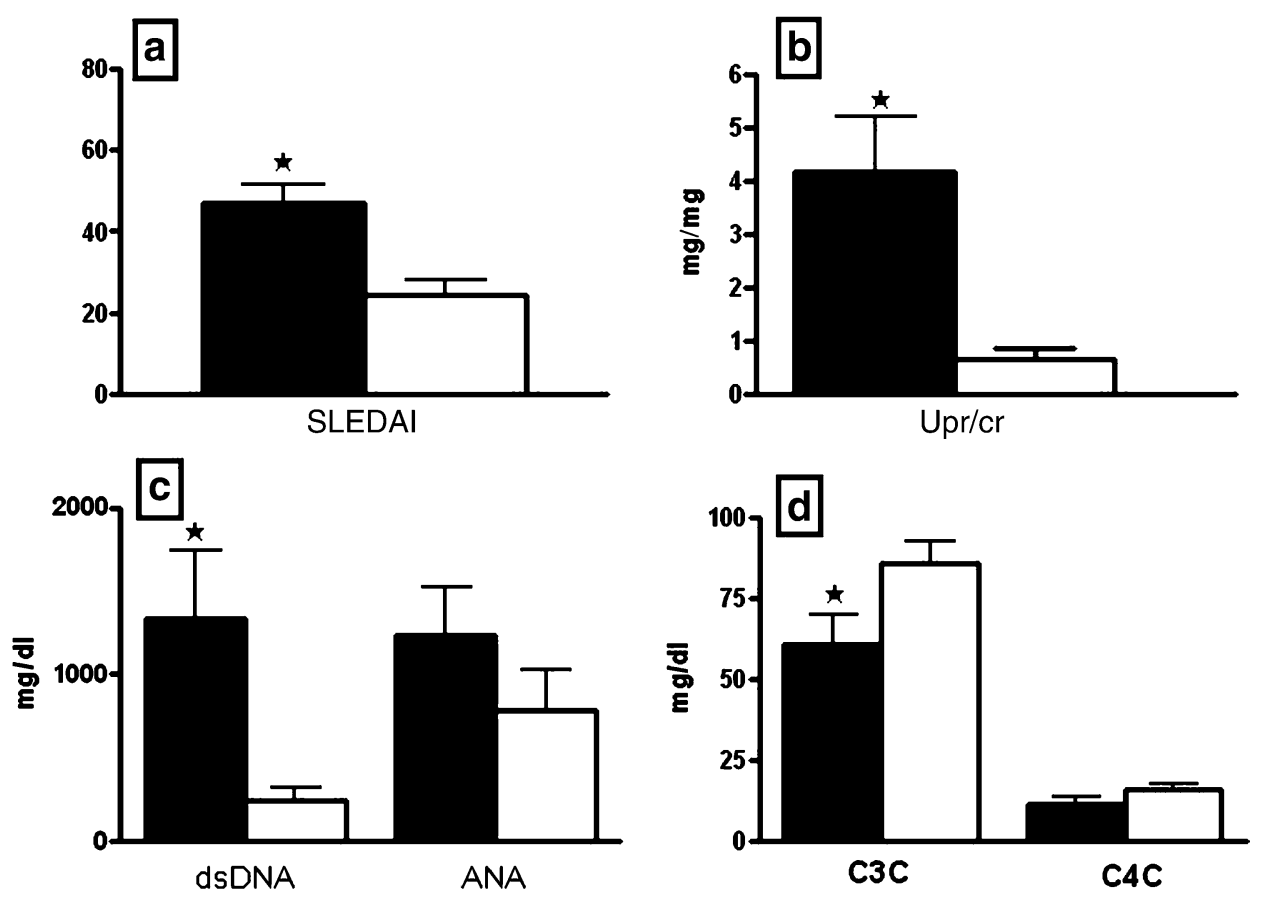

developed symptoms of cerebral vasculitis, with seizures and a cerebral infarct, demonstrated by magnetic resonance imaging (MRI) after her fourth dose of RTX. She was treated with intravenously administered gamma globulin (IVIG) and two doses of cyclophosphamide. Her neurologic deficits resolved and her vasculitis reversed, as demonstrated by MRI. She remains in remission of the lupus nephritis and cerebritis 6 months after RTX therapy.

Mild adverse reactions occurred in approximately half the patients and included pruritis, nausea, or malaise associated with the infusions. These became less when hydrocortisone was added to the pre-medication regimen and the infusion rate was slowed to 6 to 8 hours.

\section{Discussion}

To date, this report has provided the largest long-term experience in children treated with targeted B-cell depletion using the monoclonal antibody, rituximab, for severe SLE. We showed a beneficial effect of the drug in over $90 \%$ of the children in this series, which is consistent with that of previous reports [8, 19-21]. Moreover, we were able to demonstrate efficacy in controlling proteinuria in conjunction with a fall in auto-antibodies in those patients with active lupus nephritis, which mirrored that in a series of adult patients [19].

B-lymphocyte dysregulation is central in the pathogenesis of systemic lupus, with disruption of an individual's immune homeostasis. These mechanisms are multi-dimensional and are not limited to the single role of B cells in antibody production [22]. Hence, the elimination of the B-cell lineage, with potential re-population with a "healthier" line, is theoretically curative to the patient.

The safety of rituximab in young lupus patients remains unanswered. Most of the patients in our cohort tolerated the rituximab well. However, as in the European trial [11], our patients were on variable multi-drug regimens. The one patient who died did so from infectious complications in conjunction with severe immune suppression from multiple cytotoxic agents, including both rituximab and cyclophosphamide. Another young patient developed symptoms of cerebral vasculitis, with seizures and encephalopathy. The differential diagnosis included the dreaded progressive multifocal leukoencephalopathy (PML), recently reported to occur in patients treated with rituximab [23]. Although she showed clinical recovery after cyclophosphamide therapy, the case emphasizes the uncharted risks imposed by the use of drugs without well-studied indications.

The efficacy of rituximab may be short-lived in some patients. Resistance to RTX B-cell depletion may be related to antibodies against the human-mouse chimeric protein [8]. It may also be genetically influenced by polymorphisms of the FcRIIIa gene, responsible for the Fc- $\gamma$ receptor required for RTX binding to phagocytes [24]. In our series, all patients had some degree of B-cell depletion after the initial course of RTX. However, five of the 18 patients $(28 \%)$ developed clinical relapse in association with re-population of the B-cell lineage. All but one of these responded to additional courses of RTX. This is similar to recent long-term reports in a series of adults [25].

Treatment regimens in children with SLE do not conform with those of adults developed by carefully designed randomized controlled trials. This may be 
partially attributable to the fact that children $\leq 16$ years of age are excluded from these trials. This has resulted in an ambiguous and often anecdotal approach to pediatric patients, especially those with early onset and severe disease. Although intravenous cyclophosphamide treatment was established as induction therapy for proliferative lupus nephritis over two decades ago [26], its short- and long-term toxicity delayed its incorporation into pediatric regimens [27]. Paradoxically, those with severe disease have received excessive courses of toxic medications in futile efforts to preserve renal function [27]. More recently, less toxic alternative treatment regimens, including mycophenolate mofetil for both induction and maintenance therapy, have resulted in equal or improved renal survival over conventional therapies $[18,28]$. Since treatment protocols of children do not conform to conventional protocols for adults, attempts at reporting multicenter experiences are hampered by a lack of consistency in treatment regimens [11, 22]. Therefore, anecdotal center experiences such as our own are necessary in developing treatment protocols tailored towards efficacy while decreasing long-term risks on growth, fertility and malignancy.

In conclusion, RTX therapy offers a potential innovation in the current treatment regimens for children with aggressive SLE that includes both renal and extra-renal manifestations. The development of a randomized controlled treatment protocol for induction and maintenance therapy in pediatric patients is very much warranted.

Acknowledgments This study was supported, in part, by a grant from Florida's Department of Health, Children's Medical Services.

\section{References}

1. McCurdy DK, Lehman TJ, Bernstein B, Hanson V, King KK, Nadorra R, Landing BH (1992) Lupus nephritis: prognostic factors in children. Pediatrics 89:240-246

2. Contreras G, Lenz O, Pardo V, Borja E, Cely C, Iqbal K, Nahar N, de La Cuesta C, Hurtado A, Fornoni A, Beltran-Garcia L, Asif A, Young L, Diego J, Zachariah M, Smith-Norwood B (2006) Outcomes in African Americans and Hispanics with lupus nephritis. Kidney Int 69:1846-1851

3. Paredes A (2007) Can mycophenolate mofetil substitute cyclophosphamide treatment of pediatric lupus nephritis? Pediatr Nephrol 22:1077-1082

4. Opastirakul S, Chartapisak W (2005) Infection in children with lupus nephritis receiving pulse and oral cyclophosphamide therapy. Pediatr Nephrol 20:1750-1755

5. Silverman GJ (2006) Targeting of B Cells in SLE: rationale and therapeutic opportunities. Bull Hosp Jt Dis 2:51-56

6. Plosker GL, Figgitt DP (2003) Rituximab: a review of its use in non-Hodgkin's lymphoma and chronic lymphocytic leukaemia. Drugs 63:803-843

7. Edwards JC, Szczepanski L, Szechinski J, Filipowicz-Sosnowska A, Emery P, Close DR, Stevens RM, Shaw T (2004) Efficacy of
B-cell-targeted therapy with rituximab in patients with rheumatoid arthritis. N Engl J Med 350:2572-2581

8. Sfikakis PP, Boletis JN, Tsokos GC (2005) Rituximab anti-B-cell therapy in systemic lupus erythematosus: pointing to the future. Curr Opin Rheumatol 17:550-557

9. Edelbauer M, Jungraithmayr T, Zimmerhackl LB (2005) Rituximab in childhood systemic lupus erythematosus refractory to conventional immunosuppression. Pediatr Nephrol 20:811-813

10. Marks SD, Tullus K (2006) Successful outcomes with rituximab therapy with refractory childhood systemic lupus erythematosus. Pediatr Nephrol 21:598-599

11. Willems M, Haddad E, Niaudet P, Koné-Paut I, Bensman A, Cochat P, Deschênes G, Fakhouri F, Leblanc T, Llanas B, Loirat C, Pillet P, Ranchin B, Salomon R, Ulinski T, Bader-Meunier B, French Pediatric-Onset SLE Study Group (2006) Rituximab therapy for childhood-onset systemic lupus erythematosus. J Pediatr 148:623-627. Erratum in J Pediatr 2006; 149:586

12. Tan EM, Cohen AS, Fries JF (1982) The 1982 revised criteria for the classification of systemic lupus erythematosus. Arthritis Rheum 25:1271-1277

13. Gladman DD, Ibañez D, Urowitz MB (2002) Systemic lupus erythematosus disease activity index 2000. J Rheumatol 29:288291

14. Weening JJ, D’Agati VD, Schwartz MM, Seshan SV, Alpers CE, Appel GB, Balow JE, Bruijn JA, Cook T, Ferrario F, Fogo AB, Ginzler EM, Hebert L, Hill G, Hill P, Jennette JC, Kong NC, Lesavre P, Lockshin M, Looi LM, Makino H, Moura LA, Nagata M, International Society of Nephrology Working Group on the Classification of Lupus Nephritis, Renal Pathology Society Working Group on the Classification of Lupus Nephritis (2004) The classification of glomerulonephritis in systemic lupus erythematosus revisited. Kidney Int 65:521-530. Erratum in: Kidney Int 2004; 65:1132

15. Abitbol CL, Chandar J, Onder AM, Nwobi O, Montané B, Zilleruelo G (2006) Profiling proteinuria in pediatric patients. Pediatr Nephrol 21:995-1002

16. Hogg RJ, Portman RJ, Milliner D, Lemley K, Eddy A, Ingelfinger $J$ (2000) Evaluation and management of proteinuria and nephrotic syndrome in children: Recommendations from a pediatric nephrology panel established at the national kidney foundation conference on proteinuria, albuminuria, risk, assessment, detection, and elimination (PARADE). Pediatrics 105:1242 -1249

17. Schwartz GJ, Haycock GB, Edelmann CM (1976) A simple estimate of glomerular filtration in children derived from body length and plasma creatinine. J Pediatr 58:259-263

18. Ginzler EM, Dooley MA, Aranow C, Kim MY, Buyon J, Merrill JT, Petri M, Gilkeson GS, Wallace DJ, Weisman MH, Appel GB (2005) Mycophenolate mofetil or intravenous cyclophosphamide for lupus nephritis. N Engl J Med 353:2219-2228

19. Ng KP, Leandro MJ, Edwards JC, Ehrenstein MR, Cambridge G, Isenberg DA (2006) Repeated B cell depletion in treatment of refractory systemic lupus erythematosus. Ann Rheum Dis 65:942945

20. Gillis JZ, Dall'era M, Gross A, Yazdany J, Davis J (2007) Six refractory lupus patients treated with rituximab: a case series. Arthritis Rheum 57:538-542

21. Marks SD, Patey S, Brogan S, Hasson N, Pilkington C, Woo P, Tullus K (2005) B lymphocyte depletion therapy in children with refractory systemic lupus erythematosus. Arthritis Rheum $52: 3168-3174$

22. Huggins JL, Brunner HI (2006) Targeting B cells in the treatment of childhood-onset systemic lupus erythematosus. J Pediatr 148:571-573

23. Calabrese LH, Molloy ES, Huang D, Ransohoff RM (2007) Progressive multifocal leukoencephalopathy in rheumatic dis- 
eases: evolving clinical and pathologic patterns of disease. Arthritis Rheum 56:2116-2128

24. Anolik JH, Campbell D, Felgar RE, Young F, Sanz I, Rosenblatt J, Looney RJ (2003) The relationship of FcgammaRIIIa genotype to degree of $\mathrm{B}$ cell depletion by rituximab in the treatment of systemic lupus erythematosus. Arthritis Rheum 48:455-459

25. Smith KG, Jones RB, Burns SM, Jayne DR (2006) Long-term comparison of rituximab treatment for refractory systemic lupus erythematosus and vasculitis: remission, relapse, and re-treatment. Arthritis Rheum 54:2970-2982
26. Austin HA 3rd, Klippel JH, Balow JE, le Riche NG, Steinberg AD, Plotz PH, Decker JL (1986) Therapy of lupus nephritis. Controlled trial of prednisone and cytotoxic drugs. N Engl J Med 314:614-619

27. Frutos MA, Martín Gómez A, de Ramón E, Camps MT, Valera A, Garcia I, Fernandez Nebro A (2007) Intravenous cyclophosphamide in lupus nephritis: twenty years reducing dose. Nefrologia 27:12-22

28. Contreras G, Pardo V, Leclercq B, Lenz O, Tozman E, O’Nan P, Roth D (2004) Sequential therapies for proliferative lupus nephritis. N Engl J Med 350:971-980 